\title{
A Linear Manifold Color Descriptor for Medicine Package Recognition
}

\author{
Kenjiro SUGIMOTO ${ }^{\dagger * a)}$, Koji INOUE ${ }^{\dagger \dagger}$, Student Members, Yoshimitsu KUROKI ${ }^{\dagger \dagger}$, \\ and Sei-ichiro KAMATA ${ }^{\dagger b)}$, Members
}

\begin{abstract}
SUMMARY This paper presents a color-based method for medicine package recognition, called a linear manifold color descriptor (LMCD). It describes a color distribution (a set of color pixels) of a color package image as a linear manifold (an affine subspace) in the color space, and recognizes an anonymous package by linear manifold matching. Mainly due to low dimensionality of color spaces, LMCD can provide more compact description and faster computation than description styles based on histogram and dominant-color. This paper also proposes distance-based dissimilarities for linear manifold matching. Specially designed for color distribution matching, the proposed dissimilarities are theoretically appropriate more than J-divergence and canonical angles. Experiments on medicine package recognition validates that LMCD outperforms competitors including MPEG-7 color descriptors in terms of description size, computational cost and recognition rate.

key words: low-level color descriptor, linear manifold, medicine package recognition
\end{abstract}

\section{Introduction}

Dispensing error, which means incorrect prescription of medicines or dosages, is a serious social problem involving patients' and even pharmacists' lives, e.g. their lawsuits or suicides. Pharmacists have strongly demanded computerized prescription checking systems for many years since most dispensing errors are caused by human errors. Medicine package recognition using image processing technology is one of the prospective solutions for preventing from them. In order to actualize a practically useful checking system, high-speed processing without any special hardware is desired because prescribing operation should be smooth and commodity devises are easy to introduce. In view of this, we target real-time image recognition of PressThrough Package (simply called a package), a common package for pills/tablets/capsules.

Fortunately, medicine packages have discriminative elements for recognition. As Fig. 1 shows, a package contains several pills/tablets/capsules, and also some characters/symbols are regularly printed on its surface. Each pack-

Manuscript received May 2, 2011.

Manuscript revised August 20, 2011.

†The authors are with the Graduate School of Information, Production and Systems, Waseda University, Kitakyushu-shi, 8080135 Japan.

${ }^{\dagger}$ The authors are with the Kurume National College of Technology, Kurume-shi, 830-8555 Japan.

*The author is also a research fellow of Japan Society for the Promotion of Science (JSPS).

a) E-mail: sugimoto@ asagi.waseda.ac.jp

b)E-mail:kam@waseda.jp

DOI: $10.1587 /$ transinf.E95.D.1264 age is uniquely color-designed in order to prevent from visual confusing, normally showing in few distinct colors. Thus, symbol matching and color distribution matching are reasonable for package recognition. Actually, Ref. [1] recognizes character symbols printed on a package surface. However, many packages share the same symbols, e.g. the pura mark indicating plastic in Japan, and few packages are with no symbols. Its computational cost also become a problem because local information matching consumes much time in general. Therefore, this paper focuses on color distribution matching with low-level color descriptors, which provide global color description with compact size and low computational cost. This approach is significantly faster than symbol matching and could also serve as a pruning process for symbol matching.

Many color descriptors including MPEG-7 [2], [3], the international standard for video retrieval, have been proposed in the past. The most popular description style is histogram [4], [5], that is a quantized color distribution. It is easy to build and match, and also insensitive to light amount of outliers. However, the description size is relatively large. Another well-known style is dominant-color (called cluster) [6], [7]. Its size is much more compact than histogram, since it describes only their cluster properties such as centroids and variances under the assumption that a color distribution consists of several clusters. Medicine packages ba-

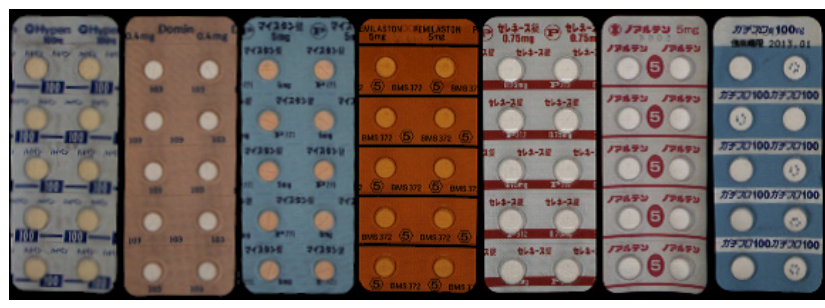

(a) Obverse side (pill-observable side)

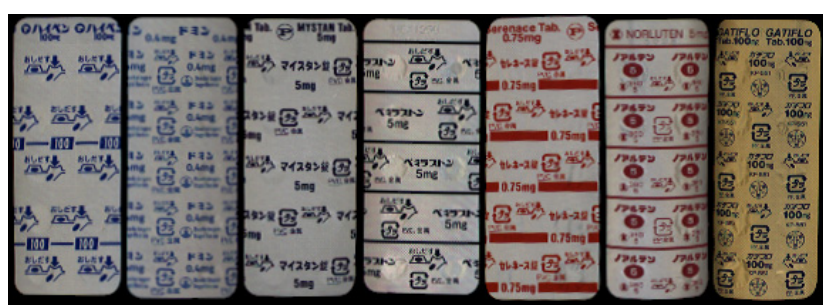

(b) Reverse side (pill-unobservable side)

Fig. 1 Examples of medicine packages called PTP. 
sically satisfy this assumption. However, clustering process to obtain the cluster properties is difficult to run fast.

This paper presents a color descriptor designed for medicine packages, called a linear manifold color descriptor (LMCD). It briefly describes a color distribution (a set of color pixels in a image) as a linear manifold (an affine subspace) in the color space. This paper also presents distance-based dissimilarities for linear manifold matching. An anonymous package can be recognized by linear manifold matching using these dissimilarities. LMCD can provide more compact description and faster computation than histogram and cluster styles. The proposed dissimilarities theoretically suit package recognition more than Jdivergence [8], canonical angles [9], [10].

The rest of the paper is organized as follows: Sect. 2 specifies a reason to employ linear manifold style, the linear manifold generation from color distributions and the dissimilarities for linear manifold matching. Section 3 discusses differences from conventional methods including $\mathrm{J}$ divergence and canonical angles. Section 4 evaluates the performance by several experiments. Finally, Sect. 5 provides our conclusions.

\section{Linear Manifold Color Descriptor}

This section explains the proposed method for medicine package recognition. Figure 2 illustrates our package matching process. First, we extract a package region from a package image registered in a database and obtain its color

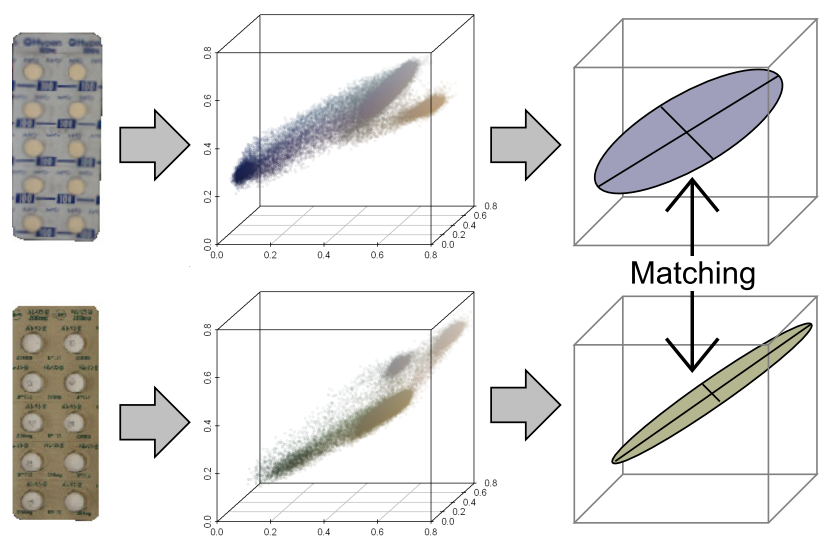

Fig. 2 Medicine packages (left), their color distributions (center) and their linear manifolds (right). distribution, that is a set of color pixels contained in the package region. Second, the color distribution is simply described as a linear manifold (an affine subspace). Third, a query package image is recognized by linear manifold matching between the query and each registered package image. Note that the proposed method generates a linear manifold from one image (a set of pixel vectors), not from an image set such as subspace methods [9], [11], [12]. The following subsections specify the main reason to employ linear manifold style, linear manifold generation, and dissimilarities between linear manifolds respectively.

\subsection{Reason to Employ Linear Manifolds}

Color distributions of medicine packages consists of few colors. Cluster style descriptors are usually applied to such a distribution. However, clustering process consumes much computer resources. Is there any workaround? The basic idea of the paper is to characterize clusters composing a distribution as a line, a plane and etc. passing through all the clusters. Consider a color distribution consisting of $C$ color clusters in $K$-dimensional color space, e.g. $K=3$ for RGB space and $K=4$ for CMYK space, where each cluster is assumed to be a dense point. Figure 3 depicts distributions with $C=1,2, \ldots, 5$ under $K=3$. If $C=1,2,3$ (Fig. $3(\mathrm{a})(\mathrm{b})(\mathrm{c}))$, we can imagine the point, the line and the plane passing through all the clusters (called passing space) respectively. Clearly, the directions of passing space express cluster configuration. If $C \geq 4$ (Fig. 3 (d)(e)), their passing spaces are three-dimensional in most cases because of limitation of $K=3$.

This approach is linked to centroid, eigenvectors and eigenvalues of distributions composed of clusters. Centroid exists at/on/in passing space and plays a role of its origin point. Eigenvectors indicate the directions of passing space. Eigenvalues express the power balance between clusters on/in passing space. On theoretical grounds, passing spaces are equivalent to affine subspaces, that is linear manifolds. As long as $C \leq K$, linear manifold can be expected to efficiently characterize the distribution. Even if $C>K$, linear manifolds still work as statistical indices. However, the more clusters, the more complicated relationship between linear manifolds and their cluster configuration. This paper concentrates on few cluster cases including color distribution of medicine packages.

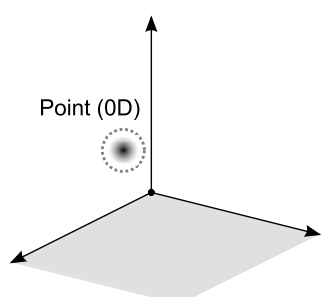

(a) $C=1$

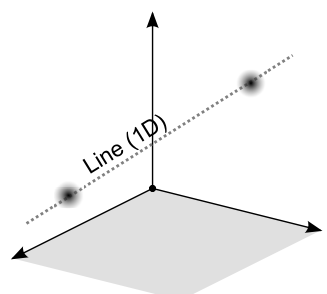

(b) $C=2$

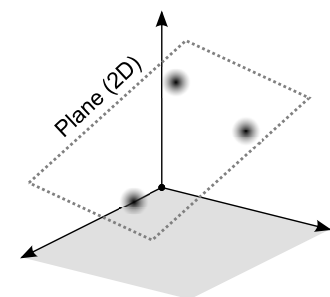

(c) $C=3$

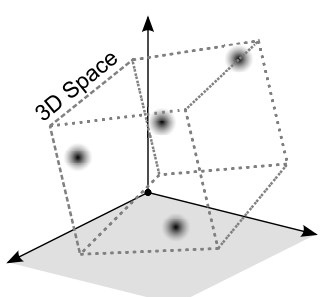

(d) $C=4$

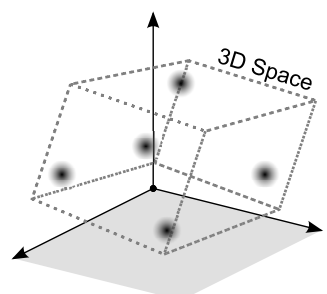

(e) $C=5$

Fig. 3 Distributions consisting of few clusters in three-dimensional space $(K=3)$. 


\subsection{Linear Manifold Generation}

Linear manifolds are generated from color distributions by using the centroid, the eigenvectors and the eigenvalues. Consider a color distribution consisting of $N$ color pixels with $K$ channels. Let $\boldsymbol{x}_{n}(n=1,2, \ldots, N)$ denote one of the $K$-dimensional vectors composing the distribution. Its covariance matrix can be expressed as

$$
\boldsymbol{S}=\frac{1}{N} \sum_{n=1}^{N}\left(\boldsymbol{x}_{n}-\overline{\boldsymbol{x}}\right)\left(\boldsymbol{x}_{n}-\overline{\boldsymbol{x}}\right)^{\top},
$$

where ${ }^{\top}$ indicates transposition of a matrix or a vector, and $\overline{\boldsymbol{x}}$ denotes the mean vector of $\boldsymbol{x}_{n}$. Eigenvalue decomposition of $S$ derives its eigenvalues $\lambda_{k}(k=1,2, \ldots, K)$ and their corresponding eigenvectors $\phi_{k}$. Note that $\lambda_{1} \geq \lambda_{2} \geq \ldots \geq$ $\lambda_{K} \geq 0$ and $\left(\boldsymbol{\phi}_{i}, \boldsymbol{\phi}_{j}\right)=\delta_{i j}(i, j=1,2, \ldots, K)$ where $(\cdot, \cdot)$ indicates an inner product and $\delta_{i j}$ denotes Kronecker delta. We define the $M$-dimensional linear manifold $(M \leq K)$ as

$$
\mathcal{P}=\left[\overline{\boldsymbol{x}} ; \boldsymbol{p}_{1}, \ldots, \boldsymbol{p}_{M}\right],
$$

where $\boldsymbol{p}_{k}=\lambda_{k}^{1 / 2} \boldsymbol{\phi}_{k}$ (called weighted eigenvectors). $M$ can be adjusted based on $C$ or according to recognition rate. Smaller $M$ actualizes stronger noise reduction in common with subspace methods [13]. This technique, which describes a color distribution as Eq. (1), is called a linear manifold color descriptor (LMCD) in the paper.

\subsection{Dissimilarities between Linear Manifolds}

This subsection entertains dissimilarities between linear manifolds. Consider two $M$-dimensional linear manifolds $\mathcal{P}=\left[\overline{\boldsymbol{x}} ; \boldsymbol{p}_{1}, \ldots, \boldsymbol{p}_{M}\right]$ and $\boldsymbol{Q}=\left[\overline{\boldsymbol{y}} ; \boldsymbol{q}_{1}, \ldots, \boldsymbol{q}_{M}\right]$, which are generated from a query image and a reference image respectively. The proposed dissimilarities are based on distance between weighted eigenvectors. The following function is introduced to deal with sign indefiniteness of eigenvectors.

$$
\delta^{2}(\boldsymbol{p}, \boldsymbol{q})=\min \left(\|\boldsymbol{p}-\boldsymbol{q}\|^{2},\|\boldsymbol{p}+\boldsymbol{q}\|^{2}\right),
$$

where $\min (\cdot)$ indicates a function which returns the minimum value of the arguments.

\subsubsection{Distance between Centroids with Matric Matrix}

The first proposed dissimilarity is the distance between centroids in differential linear manifold.

$$
d^{2}(\mathcal{P}, Q)=(\overline{\boldsymbol{x}}-\overline{\boldsymbol{y}})^{\top} \boldsymbol{D}^{\top} \boldsymbol{D}(\overline{\boldsymbol{x}}-\overline{\boldsymbol{y}}),
$$

where $\boldsymbol{D}=\left[\boldsymbol{p}_{1}-\boldsymbol{q}_{1}, \boldsymbol{p}_{2}-\boldsymbol{q}_{2}, \ldots, \boldsymbol{p}_{r}-\boldsymbol{q}_{r}\right] . \boldsymbol{D}^{\top} \boldsymbol{D}$ indicates a metric matrix of Riemann space. Equation (3) returns 0 if either the centroids or the weighted eigenvectors is the same as each other.

\subsubsection{Diagonal Pairing}

The second dissimilarity is the summation of $M$ weighted eigenvector pairs. Its calculation time is in $O(K M)$.

$$
d^{2}(\mathcal{P}, Q)=\sum_{k=1}^{M} \delta^{2}\left(\boldsymbol{p}_{k}, \boldsymbol{q}_{k}\right) .
$$

If $\boldsymbol{p}_{k}$ or $\boldsymbol{q}_{k}$ is sign-reversed in advance such that $\delta^{2}\left(\boldsymbol{p}_{k}, \boldsymbol{q}_{k}\right)=$ $\left\|\boldsymbol{p}_{k}-\boldsymbol{q}_{k}\right\|^{2}$, Eq. (4) can be rewritten as

$$
d^{2}(\mathcal{P}, \boldsymbol{Q})=\|\boldsymbol{D}\|_{\mathrm{F}}^{2}=\operatorname{tr}\left(\boldsymbol{D}^{\top} \boldsymbol{D}\right),
$$

where and $\|\cdot\|_{\mathrm{F}}$ indicates Frobenius norm of a matrix.

\subsubsection{Minimum Cost Pairing}

The third proposed dissimilarity is calculated with minimization of the dissimilarity cost. Equation (4) might return an improper value if some eigenvalues switch their places because of noise influence. Although the weighted eigenvectors are paired in descending order of eigenvalues, eigenvectors are unordered intrinsically. A solution to the pairing problem is to search all the pairs for the minimum dissimilarity. Let $B=\left\{b_{1}, b_{2}, \ldots, b_{M !}\right\}(|B|=M !)$ denote a set of possible pairs, e.g. $b_{1}=\{(0,0),(1,1),(2,2)\}$ represents Eq. (4). The dissimilarity for the weighted eigenvectors can be expressed as

$$
d^{2}(\mathcal{P}, Q)=\min _{b \in B} \sum_{(\boldsymbol{p}, \boldsymbol{q}) \in b} \delta^{2}(\boldsymbol{p}, \boldsymbol{q})
$$

The pairing problem is identical to a minimum matching problem of weighted bipartite graphs. Two typical solutions are well-known: full search in exponential time $O(K M !)$ and Hungarian algorithm [14] in polynomial time $O\left(K M^{4}\right)$ and $O\left(K M^{3}\right)$. In the case of low-dimensional space such as color space, full search is expected to be faster and easier to implement than Hungarian algorithm, which could be more effective if $M$ is large.

\subsubsection{Round-Robin}

Another more simple countermeasure against unordered eigenvectors is round-robin, that is the summation of the distance between all the pairs.

$$
d^{2}(\mathcal{P}, Q)=\sum_{i=1}^{M} \sum_{j=1}^{M} \delta^{2}\left(\boldsymbol{p}_{i}, \boldsymbol{q}_{j}\right) .
$$

The calculation time is clearly in $O\left(K M^{2}\right)$.

\section{Discussion about Conventional Methods}

This section introduces conventional methods based on linear manifold or linear subspace matching, and clarifies differences from LMCD. 


\subsection{Kullback-Leibler Divergence}

Kullback-Leibler (KL) divergence [15] is a well-known measure between two probability density functions. KL divergence between two multivariate normal distributions can be utilized as a dissimilarity between linear manifolds [12]. This is because liner manifolds derived by principal component analysis can be theoretically regarded as an approximation of a multivariate normal distribution. Here, focus on J-divergence [8] (also called symmetric KL divergence), a modification bringing symmetry as distance-like measures:

$$
\begin{aligned}
d(\mathcal{P}, Q)= & (\overline{\boldsymbol{x}}-\overline{\boldsymbol{y}})^{\top}\left(\boldsymbol{S}_{p}^{-1}+\boldsymbol{S}_{q}^{-1}\right)(\overline{\boldsymbol{x}}-\overline{\boldsymbol{y}}) \\
& +\operatorname{tr}\left(\boldsymbol{S}_{p}^{-1} \boldsymbol{S}_{q}\right)+\operatorname{tr}\left(\boldsymbol{S}_{q}^{-1} \boldsymbol{S}_{p}\right)
\end{aligned}
$$

where $\boldsymbol{S}_{p}$ and $\boldsymbol{S}_{q}$ are the covariance matrices of $\mathcal{P}$ and $Q$ respectively, and redundant coefficients/terms are abbreviated.

J-divergence is imperfect for color distribution matching. Substituting $\overline{\boldsymbol{x}}=\overline{\boldsymbol{y}}$ for Eq. (7) results in

$$
d(\mathcal{P}, \boldsymbol{Q})=\operatorname{tr}\left(\boldsymbol{\Lambda}_{p}^{-1} \boldsymbol{\Lambda}_{q}\right)+\operatorname{tr}\left(\boldsymbol{\Lambda}_{q}^{-1} \boldsymbol{\Lambda}_{p}\right),
$$

where $\boldsymbol{\Lambda}_{p}$ and $\boldsymbol{\Lambda}_{q}$ are the diagonal eigenvalue matrices of $\mathcal{P}$ and $\boldsymbol{Q}$. Obviously, Eq. (8) disregards eigenvectors. This is a fatal information loss because eigenvectors are still useful even if $\overline{\boldsymbol{x}}=\overline{\boldsymbol{y}}$. Incidentally, this loss occurs also in KL divergence between two multivariate normal distributions. On the other hand, the proposed dissimilarities utilize eigenvectors for matching even if $\overline{\boldsymbol{x}}=\overline{\boldsymbol{y}}$.

\subsection{Mutual Subspace Method}

The mutual subspace method (MSM) [9] is a successful technique on pattern recognition. It describes a set of pattern vectors as a linear subspace and recognize a query pattern set by subspace matching. MSM and LMCD are seemingly similar in view of linear and affine subspaces. Nevertheless, MSM is unfit to color distribution matching.

The mechanism of MSM is as follows: MSM requires pattern vectors belonging to a category to locate in a linear subspace. Consider an extremely simplified subspace, that is a line passing through the origin. MSM also requires each category line to locate to a unique direction. The dissimilarity between two categories is calculated as the angle between the two category lines. The angle is called canonical angle.

First, color distributions cannot satisfy the requirements because all the clusters composing a distribution do not locate on a linear subspace passing through the origin (namely black) in general. Even if the centroid is translated to the origin, the clusters locating at various directions could not be accurately approximated as a low-dimensional subspace. Thus, linear subspaces cannot describe color clusters efficiently.

Second, MSM treats $\phi_{k}$ only and disregards $\lambda_{k}$. This is because $\lambda_{k}$ (variance) is unreliable as a statistical index on character or face recognition. More samples provide more reliable statistical indices. However, a distribution of character or face pattern vectors is composed of at most 100 pattern vectors. This is not enough to rely on $\lambda_{k}$. By contrast, a color distribution consists of at least 10000 pixel vectors. Thus, $\lambda_{k}$ is a reliable index on color distribution matching.

\section{Performance Evaluation}

This section evaluates the performance of LMCD in terms of recognition accuracy, robustness, description size and calculation time. The test environment mounts Intel Core2 Quad 2.33 GHz CPU with $2 \mathrm{~GB}$ main memory. The competitors are (i) RGB/HSV histograms quantized into $2 \times 2 \times 2$, $4 \times 4 \times 4$ and $8 \times 8 \times 8$ bins, (ii) the scalable color descriptor (SCD) in MPEG-7, a histogram-style one, (iii) the dominant color descriptor (DCD) in MPEG-7, a cluster-style one, (iv) J-divergence, (v) canonical angles and (vi) LMCD. The histograms and SCD are matched by the nearest neighbor search using the histogram intersection [16] and Euclid distance respectively. We use the MPEG-7 descriptor implementation by Lux and Chatzichristofis [17]-[19] and selfproduced implementations for the others.

Table 1 lists three test sets used for the experiments where \#Category indicates the total number of package categories and \#Image indicates the total number of package images. OBV contains obverse side image (Fig. 1 (a)), REV contains reverse ones (Fig. 1 (b)), and ALL is simply the mix of OBV and REV. Note that ALL treats the obverse/reverse sides of a package as two different categories in order to increase the number of categories because more categories contributes to more severe experiments. Each test set is equally-divided into two subsets for training and matching phases. All the test images are 24-bits RGB and their size is roughly from QVGA $(320 \times 240$ pixels $)$ to VGA $(640 \times 480$ pixels). Although each image captures one whole package under the almost same light condition, some of the packages provide highlight or shadow regions owing to metallic surface or package bend respectively. The package region in each image is extracted in advance.

\subsection{Recognition Accuracy}

Table 2 lists the recognition rate of the competitors. "LMCD-Eq. (3)" means LMCD using the dissimilarity of Eq. (3). The bold numbers in the table are the best rate in $2 \times 2 \times 2,4 \times 4 \times 4$ and $8 \times 8 \times 8$ bins for the histograms or in $M=1,2,3$ for LMCD for each test set of each method. These results reveal the following facts:

- Comparison between LMCD-Eq. (3) with $\boldsymbol{D}^{\top} \boldsymbol{D}=\boldsymbol{I}$, i.e. Euclid distance between centroids, and LMCDEq. (3) shows the effect of a metric matrix $\boldsymbol{D}^{\top} \boldsymbol{D}$. Even

Table 1 Three test sets for performance evaluation.

\begin{tabular}{|c|r|r|l|}
\hline Test Set & \#Category & \#Image & Note \\
\hline OBV & 84 & 900 & Pill-observable \\
REV & 84 & 936 & Pill-unobservable \\
ALL & 168 & 1836 & OBV+REV \\
\hline
\end{tabular}


Table 2 Recognition rate [\%].

\begin{tabular}{|l|ccc|ccc|ccc|}
\hline & \multicolumn{3}{|c|}{ OBV } & \multicolumn{3}{c|}{ REV } & \multicolumn{3}{c|}{ ALL } \\
& $2 \times 2 \times 2$ & $4 \times 4 \times 4$ & $8 \times 8 \times 8$ & $2 \times 2 \times 2$ & $4 \times 4 \times 4$ & $8 \times 8 \times 8$ & $2 \times 2 \times 2$ & $4 \times 4 \times 4$ & $8 \times 8 \times 8$ \\
\hline RGB histogram & 59.11 & 83.56 & $\mathbf{9 5 . 3 3}$ & 47.44 & 80.13 & $\mathbf{8 1 . 6 2}$ & 49.35 & 78.98 & $\mathbf{8 8 . 0 2}$ \\
HSV histogram & 61.56 & 89.11 & $\mathbf{9 5 . 5 6}$ & 35.47 & 71.80 & $\mathbf{8 4 . 1 9}$ & 42.92 & 79.74 & $\mathbf{8 9 . 6 5}$ \\
\hline \hline & $M=1$ & $M=2$ & $M=3$ & $M=1$ & $M=2$ & $M=3$ & $M=1$ & $M=2$ & $M=3$ \\
\hline SCD [2], [3] & & 98.67 & & & 86.75 & & & 92.37 & \\
J-divergence [8], Eq. (7) & & 100.00 & & & 89.74 & & & 94.77 & N/A \\
Canonical angles [9], [11] & $\mathbf{8 2 . 0 0}$ & 59.78 & N/A & $\mathbf{7 7 . 7 8}$ & 15.60 & N/A & $\mathbf{6 8 . 6 3}$ & 29.85 & $/$ ALL \\
\hline LMCD-Eq. (3) with $\boldsymbol{D}^{\top} \boldsymbol{D}=\boldsymbol{I}$ & & 58.89 & & & 50.86 & & & 48.80 & \\
LMCD-Eq. (3) & 56.00 & 88.44 & $\mathbf{8 9 . 3 3}$ & 54.70 & 74.57 & $\mathbf{7 9 . 0 6}$ & 45.31 & 76.58 & $\mathbf{8 1 . 2 6}$ \\
LMCD-Eq. (4) with $\lambda_{k}=1$ & 82.00 & $\mathbf{8 4 . 4 4}$ & 83.11 & $\mathbf{7 7 . 7 8}$ & 36.75 & 35.04 & $\mathbf{6 8 . 6 3}$ & 54.58 & 53.16 \\
LMCD-Eq. (4) & 92.89 & $\mathbf{9 9 . 3 3}$ & 99.11 & 92.31 & $\mathbf{9 3 . 5 9}$ & 92.52 & 89.00 & $\mathbf{9 5 . 1 0}$ & 94.77 \\
LMCD-Eq. (5) & 92.89 & $\mathbf{9 9 . 3 3}$ & $\mathbf{9 9 . 3 3}$ & 92.31 & $\mathbf{9 2 . 5 2}$ & 91.67 & 89.00 & $\mathbf{9 4 . 5 5}$ & 94.44 \\
LMCD-Eq. (6) & 92.89 & 99.56 & $\mathbf{9 9 . 7 8}$ & 92.31 & 92.95 & $\mathbf{9 3 . 1 6}$ & 89.00 & 95.21 & $\mathbf{9 5 . 7 5}$ \\
\hline
\end{tabular}

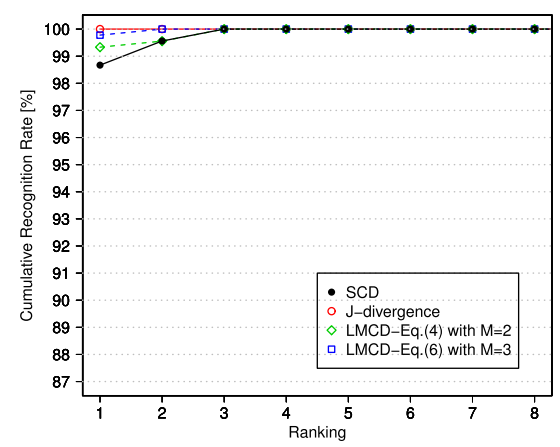

(a) $\mathrm{OBV}$

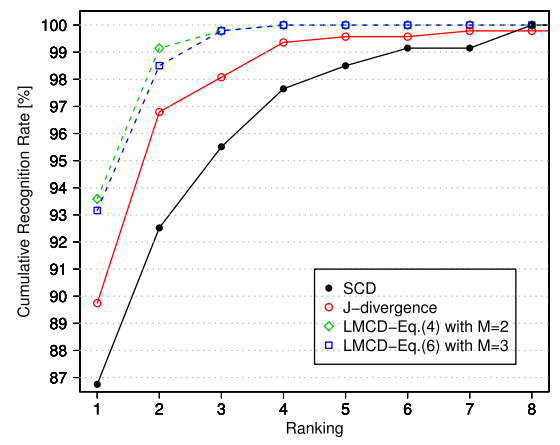

(b) REV

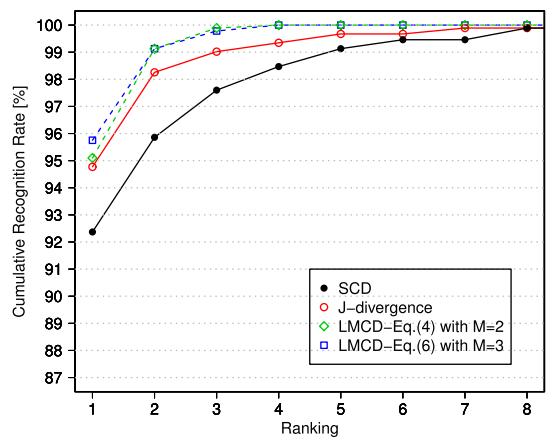

(c) ALL

Fig. 4 Cumulative recognition rate [\%].

though the metric matrix can raise the rate drastically, the rate cannot reach to that of LMCD-Eq. (4).

- Centroid information are essentially sensitive to brightness change as we expected in Sec. 2.3. Since the packages are especially metallic, the rate is relatively worse. Incidentally, the simple sum of Eq. (3) and Eq. (4) cannot surpass the rate of LMCD-Eq. (3).

- Comparison between canonical angles and LMCDEq. (4) with $\lambda_{k}=1$ supports efficiency of the proposed dissimilarity against canonical angles. LMCD-Eq. (4) with $\lambda_{k}=1$ achieves higher rate than canonical angles at $M=2,3$. Canonical angles are rather inaccurate at $M=2$ and impossible to use $M=3$.

- Comparison between LMCD-Eq. (4) with $\lambda_{k}=1$ and LMCD-Eq. (4) validates that weighted eigenvectors are efficient for color distribution matching. LMCDEq. (4) shows more than 50\% higher rate than LMCDEq. (4) with $\lambda_{k}=1$ at $M=2$ in REV.

- LMCD-Eq. (5), which minimizes dissimilarity by paring search, shows slightly lower rate than LMCDEq. (4). In fact, minimization is unnesessary because no pair mismatch occurs by LMCD-Eq. (4) in the test sets. The rate decreasing may be attributed to dissimilarity depression of incorrect categories.

- Clearly, LMCDs are more accurate than RGB/HSV histograms.

Next, Fig. 4 plots the cumulative recognition rate of SCD, J- divergence, LMCD-Eq. (4) with $M=2$ and LMCD-Eq. (6) with $M=3$, which are the methods showing top-level rate in Table 2. The two LMCDs are comparable in OBV and superior in REV and ALL to SCD and J-divergence.

\subsection{Analysis on Incorrect Answers}

Given a query image, matching phase answers the categories nearest to the query (called candidates). Table 3 lists four cases of answering incorrect categories by LMCD-Eq. (4). For each row, the left image is a query and the four right images are from first candidate to fourth candidate for the query. The number shown below in each category indicates the dissimilarity between the corresponding query and the category prototype. Apparently, each query and the four candidates share their dominant colors, e.g. they share the sheet, the pill and even the symbol colors. It is difficult even for people to visually distinguish them instantaneously. Conversely, these results demonstrate that LMCD can accurately distinguish categories with distinct colors.

\subsection{Robustness Tests}

Robustness is also an important factor for color descriptors. It can be quantified as the dissimilarity between an original image and one of its operated images. Figure 5 shows the robustness test results of LMCD-Eq. (4) to rotation, scale 
Table 3 Query images and their incorrect answers (the correct one is in boldface).

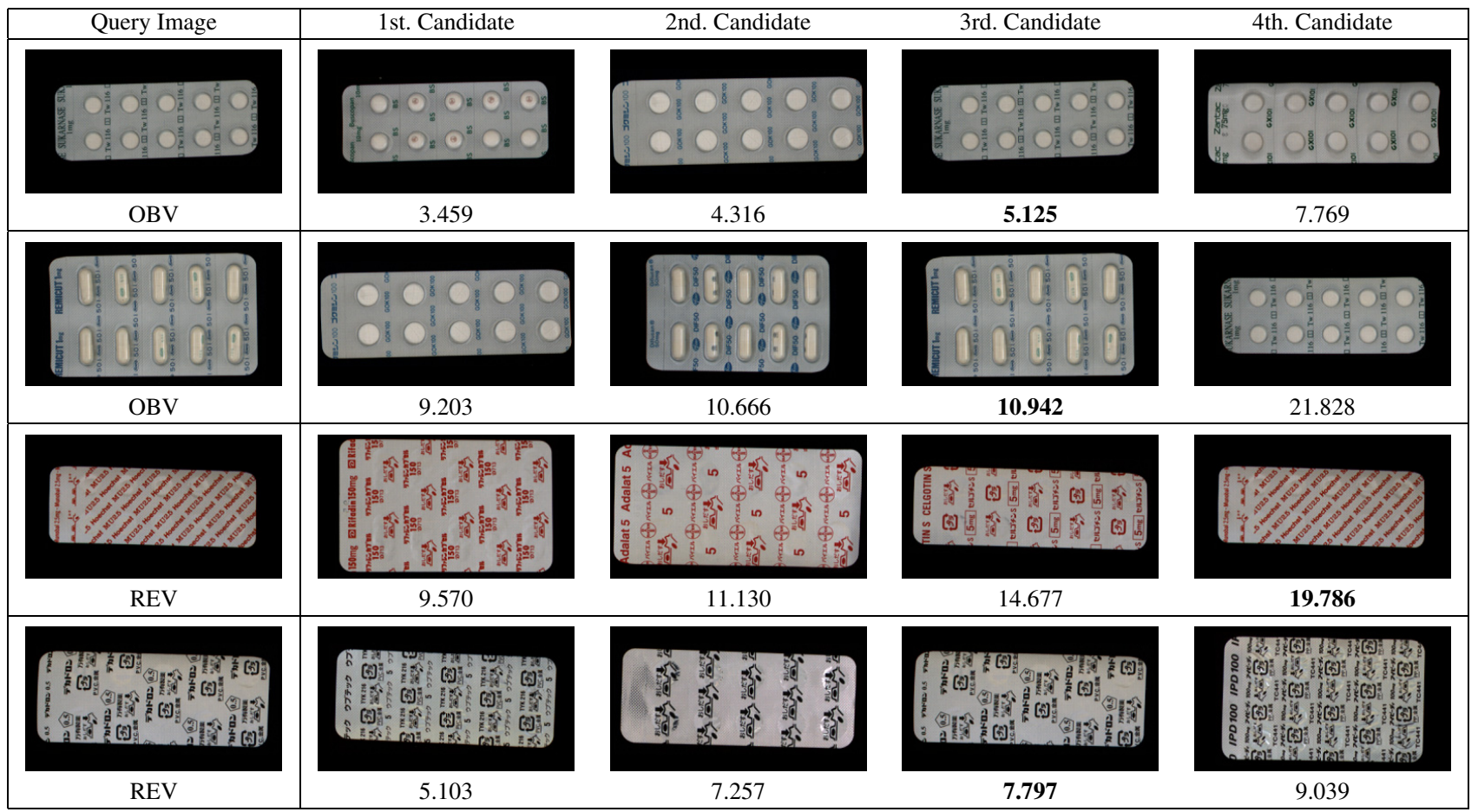

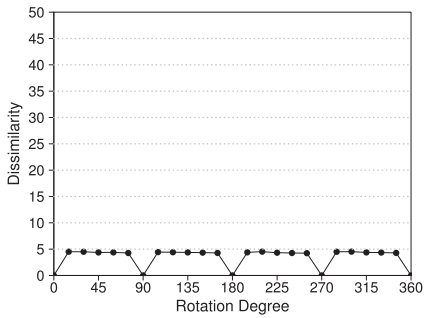

(a) Rotation

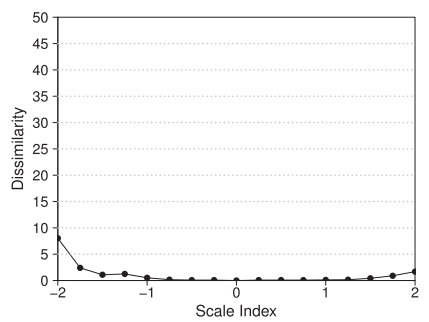

(b) Scale change

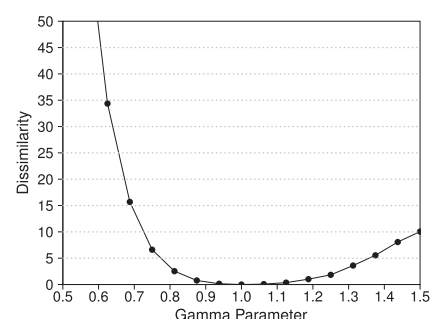

(c) Gamma correction

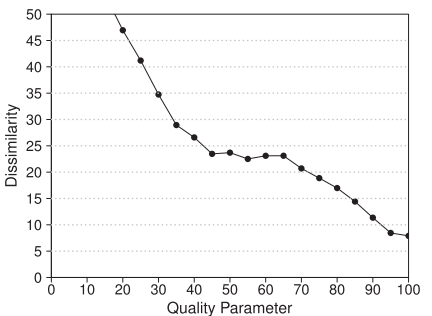

(d) JPEG compression

Fig. 5 The dissimilarity between an original image and one of its operated images.

change, gamma correction and JPEG compression. The scale index indicates size scaling by $2^{\text {(ScaleIndex) }} \times 2^{\text {(ScaleIndex) }}$.

The results reveal that LMCD is robust to rotation and scale change but variant to gamma correction and JPEG compression. In rotation and scale change, amount of dissimilarity change is consistently small, because LMCD disregards pixel position in images and scale change does not change the shape of color distributions. In gamma correction and JPEG compression, the amount is comparativelylarge, since the two operations directly affect color information, e.g. JPEG compression changes pixel color by quantizing color distribution to reduce file size.

Robustness to reflection under real situations should be also considered. LMCD-Eq. (4) appears to slightly tolerate change of gamma parameter in Fig. 5 (c). This is because disregarding centroids in Eq. (4) probably produces a tolerability of light-dark change over the entire image such as Gamma correction. Next, highlight regions arise particularly on metallic surface such as medicine packages. As far as we can predict, LMCD is easily influenced by white high-
Table 4 Size and calculation time [ms/package].

\begin{tabular}{|l|c|rr|}
\hline Descriptor & Size & Training & Matching \\
\hline RGB histogram & $8 \times 8 \times 8=512$ & 1.20 & 1.85 \\
HSV histogram & $8 \times 8 \times 8=512$ & 2.95 & 3.72 \\
SCD (histogram) & $8 \times 8 \times 4=256$ & 6.85 & 7.04 \\
DCD (cluster) & $K C$ at least & 374.05 & 245.21 \\
MSM & $K^{2}$ at most & 1.70 & 2.72 \\
LMCD-Eq. (4) & $K^{2}+K$ at most & 1.70 & 1.79 \\
\hline
\end{tabular}

lights since white strongly pulls eigenvectors from a corner in RGB color space. Intuitively, robust regression algorithms could ease reflection problem. Further validations are desirable in the future.

\subsection{Description Size and Calculation Time}

Table 4 indicates the description size of RGB/HSV histograms, SCD, DCD, MSM and LMCD. MSM and LMCD is the most compact in them. The histograms including $\mathrm{SCD}$ requires many elements to store. The other methods 
require far fewer elements than SCD. The size of DCD is proportional to $C$ and usually larger than MSM and LMCD. MSM and LMCD requires just nine and twelve elements in RGB case respectively. Note that the degree-of-freedom of LMCD is smaller than its size owing to orthogonality conditions between eigenvectors (c.f. Cholesky decomposition).

Table 4 also indicates their training and matching time. Interestingly, LMCD shows the processing time comparable to RGB histogram and shorter than HSV histogram because of color convert. Many histogram elements cause slow matching. DCD is severely slower than the others since clustering process runs heavy iterative computation. The others are fast sufficiently to run in real-time. Matching of MSM is nearly 1 [ms/package] slower than that of LMCD because of eigenvalue decomposition for each matching. This result validates that LMCD can be calculated as fast as histograms can.

\subsection{The Number of Clusters and Dimensions}

The relationship between $C$ and $M$, as mentioned in Sec. 2.1, can be examined also from Table 2. OBV shows higher rate than REV in every case because obverse side shows in more colors than reverse side in most cases thanks to pill colors. More colors cause easier category separation. Most obverse and reverse side images in the test sets show in two and three colors respectively. According to the fact that an $M$-dimensional linear manifold can efficiently characterize $(M+1)$ clusters, $M=2$ for OBV and $M=1$ for REV are supposed to be optimum. LMCD-Eq. (4) and $\lambda_{k}=1$ actually shows the best rate at the parameters in the both sets. The rate difference between $M=1,2$ additionally supports our analysis. For example, in the case of LMCD-Eq. (4), the difference in OBV $(99.33-92.89=6.44 \%)$ is significantlylarger than that in REV $(93.59-92.31=1.28 \%)$. This fact indicates that the second eigenvector is more meaningful for OBV than for REV.

\section{Conclusions}

This paper has presented a color-based method for medicine package recognition, called LMCD. It simply describes a color distribution as a linear manifold. We have clarified the superiority of linear manifolds and distance-based dissimilarities on color distribution matching by theoretically comparing with MSM and J-divergence. Our experiments revealed that LMCD clearly outperformed competitors in terms of recognition accuracy, description size and training/matching time. Surprisingly, the processing speed was comparable to RGB histograms.

The experiments have also resulted in supporting our initial idea: an $M$-dimensional linear manifold can characterize $(M+1)$ clusters. This requirement is slightly limited and LMCD might be difficult to run in real environments because of widely variable light condition and camera parameter. However, as long as environments satisfying the assumption, LMCD can provide much more compact de- scription and much faster computation than the conventional color descriptors.

\section{Acknowledgments}

This work was supported by the Grant-in-Aid for Japan Society for the Promotion of Science (JSPS).

\section{References}

[1] H. Kameyama, Japan Patent Kokai 2005-034479, Feb. 102005.

[2] T. Sikora, "The MPEG-7 visual standard for content description an overview," IEEE Trans. Circuits Syst. Video Technol., vol.11, no.6, pp.696-702, June 2001.

[3] B.S. Manjunath, J.R. Ohm, V.V. Vasudevan, and A. Yamada, "Color and texture descriptors," IEEE Trans. Circuits Syst. Video Technol., vol.11, no.6, pp.703-715, June 2001.

[4] J. Hafner, H.S. Sawhney, W. Equitz, M. Flickner, and W. Niblack, "Efficient color histogram indexing for quadratic form distance functions," IEEE Trans. Pattern Anal. Mach. Intell., vol.17, no.7, pp.729-736, July 1995.

[5] L.V. Tran and R. Lenz, "PCA-based representation of color distributions for color-based image retrieval," Proc. IEEE ICIP, vol.2, pp.697-700, Oct. 2001.

[6] Y. Deng, B.S. Manjunath, C. Kenney, M. Moore, and H. Shin, "An efficient color representation for image retrieval," IEEE Trans. Image Process., vol.10, no.1, pp.140-147, Jan. 2001.

[7] N.C. Yang, W.H. Chang, C.M. Kuo, and T.H. Li, "A fast MPEG7 dominant color extraction with new similarity," J. Vis. Commun. Image Represent., vol.19, pp.92-105, Feb. 2008.

[8] A. Cichocki and S. Amari, "Families of alpha- beta- and gammadivergences: flexible and robust measures of similarities," Entropy, vol.12, no.6, pp.1532-1568, June 2010.

[9] K. Maeda and S. Watanabe, "A pattern matching method with local structure," IEICE Trans. Inf. \& Syst. (Japanese Edition), vol.J68-D, no.3, pp.345-352, March 1985.

[10] F. Chatelin, Eigenvalues of matrices, 1st ed., John Wiley \& Sons, 1993.

[11] K. Fukui, O. Yamaguchi, K. Suzuki, and K. Maeda, "Face recognition under variable lighting condition with constrained mutual subspace method: Learning of constraint subspace to reduce influence of lighting changes," IEICE Trans. Inf. \& Syst. (Japanese Edition), vol.J82-D-II, no.4, pp.613-620, April 1999.

[12] J.W.F.G. Shakhnarovich and T. Darrell, "Face recognition from long-term observations," Proc. IEEE ECCV, pp.851-868, 2002.

[13] E. Oja, Subspace methods of pattern recognition, Research Studies Press, 1983.

[14] R. Burkard, M. Dell'Amico, and S. Martello, Assignment Problems, Society for Industrial and Applied Mathematics, 2009.

[15] S. Kullback and R. Leibler, "On information and sufficiency," Ann. of Mathematical Statistics, vol.22, no.1, pp.79-86, 1951.

[16] M.J. Swain and D.H. Ballard, "Color indexing," Int. J. Comput. Vis., vol.7, no.1, pp.11-32, Nov. 1991.

[17] "Image processing and retrieval trends: MPEG-7 descriptors." http://savvash.blogspot.com/p/mpeg-7-descriptors.html.

[18] M. Lux and S.A. Chatzichristofis, "LIRe: Lucene image retrieval an extensible java CBIR library," Proc. ACM Int'l Conf. Multimedia, pp.1085-1087, Oct. 2008.

[19] S.A. Chatzichristofis, Y.S. Boutalis, and M. Lux, "Img(Rummager): An interactive content based image retrieval system," Proc. IEEE Computer Soc., pp.151-153, Aug. 2009. 


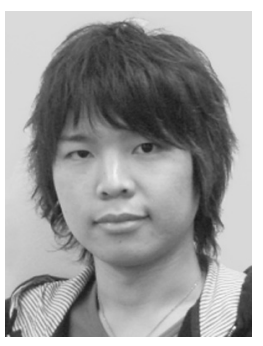

Kenjiro Sugimoto received the B.E. and M.E. degrees in 2007 and 2009 from Kurume National College of Technology, Japan, and Waseda University, Japan, respectively. He is currently pursuing a Ph.D. degree at Graduate School of Information, Production and Systems, Waseda Univeristy, and has been a Research Fellow of the Japan Society for the Promotion of Science (JSPS) since 2010. His current research interests are in image compression and pattern recognition. He is a student member of

IEEE, ACM, IPSJ and IIEEJ.

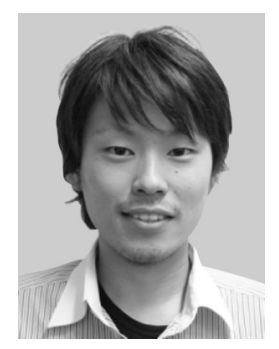

Koji Inoue is currently working toward the diploma in Department of Control and Information Systems Engineering, Kurume National College of Technology. His research interests include pattern recognition and image processing.

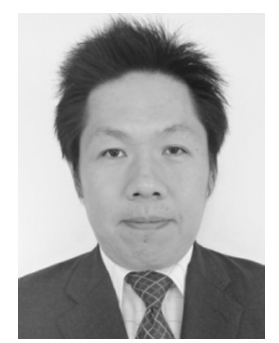

Yoshimitsu Kuroki received the B.E., M.E., and Ph.D. in Electronic Engineering from Kyushu Institute of Technology, Kitakyushu, Japan, in 1991, 1993, and 1996, respectively. From 1996 to 2001, he worked in the Department of Electrical Engineering, Kagoshima National College of Technology. Since 2001, he has been an associate professor in the Department of Control and Information Systems Engineering, Kurume National College of Technology. His research interests include image compression, image processing, and pattern recognition. He is a member of IEEE, IPSJ, ITE and IIEEJ.

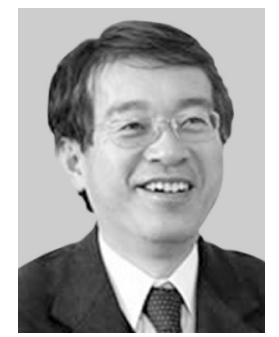

Sei-ichiro Kamata received the M.S. degree in computer science from Kyushu University, Fukuoka, Japan, in 1985, and the Doctor of Computer Science, Kyushu Institute of Technology, Kitakyushu, Japan, in 1995. From 1985 to 1988, he was with NEC, Ltd., Kawasaki, Japan. In 1988, he joined the faculty at Kyushu Institute of Technology. From 1996 to 2001, he has been an Associate Professor in the Department of Intelligent System, Graduate School of Information Science and Electrical Engineering, Kyushu University. Since 2003, he has been a professor in the Graduate School of Information, Production and Systems, Waseda University. In 1990 and 1994, he was a Visiting Researcher at the University of Maine, Orono. His research interests include image processing, pattern recognition, image compression, and space-filling curve application. He is a member of IEEE and ITE in Japan. 